\title{
Relação da composição corporal e aptidão física de idosos praticantes de musculação
}

\author{
Márcio Junior Strassburger", Simone Zeni Strassburger*", Alana Bortolan Sacon "**, Cândida Grassi ${ }^{* * * *}$
}

\section{Resumo}

A população brasileira está em processo de envelhecimento e o exercício físico tem muito a contribuir, mantendo o organismo saudável e reduzindo os efeitos deletérios do envelhecimento. Este artigo objetivou verificar a antropometria e a aptidão física em idosos praticantes de musculação. Trata-se de um estudo transversal, que apresenta e discute os dados da avaliação de 43 idosos, praticantes dessa atividade, quanto à composição corporal, flexibilidade e força de membros superiores e inferiores. O tempo médio de academia foi de 70,67 $\pm 100,98$ meses, uma frequência semanal de $3,04 \pm 1,02$ vezes. O sobrepeso foi o mais encontrado, em $46,51 \%$ dos idosos, e mais frequente nos homens. As mulheres apresentam melhores resultados na flexibilidade, enquanto os homens apresentam melhores resultados para os testes de força. De modo geral, o tempo de academia não interfere na composição corporal, porém os homens com sobrepeso têm menor tempo de/na academia enquanto as mulheres com sobrepeso têm maior tempo de academia. Verifica-se, no grupo avaliado, que a musculação não está proporcionando grandes alterações na aptidão física e na antropometria. Salienta-se que essa modalidade está cada vez mais difundida por essa população, revelando à necessidade de mais estudos na área, para a percepção dos reais efeitos desse treino no processo de envelhecimento.

Palavras-chave: Exercício. Antropometria. Envelhecimento.

\section{Introdução}

O crescimento no número de idosos é um fenômeno mundial, no Brasil, essas modificações estão ocorrendo de forma radical e acelerada (VERAS, 2009; VERAS, 2003). A velhice não é mais privilégio de poucos, é um fenômeno natural e social, único, indivisível, que se depara com problemas e limitações biológicas,

* Fisioterapeuta. Especialista em Fisiologia do Exercício. Mestre em saúde coletiva. Docente do curso de Fisioterapia da Universidade Regional do Noroeste do Estado do Rio Grande do Sul (Unijuí). Endereço para correspondência: Rua Janne Thorstemberg, 220, Morada do Sol, CEP: 98700-000, Ijuí(RS). E-mail: marcio.s@unijui.edu.br

** Fisioterapeuta. Especialista em Fisioterapia Neurológica. Mestre e doutoranda em saúde da criança. Docente do curso de Fisioterapia da Universidade Regional do Noroeste do Estado do Rio Grande do Sul (Unijuí). E-mail: simone.s@unijui.edu.br

**** Fisioterapeuta. Graduada na Universidade Regional do Noroeste do Estado do Rio Grande do Sul (Unijuí). E-mail: alanasacon@yahoo.com.br

***** Fisioterapeuta. Graduada na Universidade Regional do Noroeste do Estado do Rio Grande do Sul (Unijuí). E-mail: candida_grassi@yahoo.com.br

$\rightarrow$ http://dx.doi.org/10.5335/rbceh.2013.2662 
econômicas e socioculturais, ocasionando a deterioração de um organismo maduro. Essas mudanças iniciam-se após a maturação sexual e podem levar à redução da capacidade de resposta dos indivíduos ao estresse ambiental e à manutenção da homeostasia, dentre outras mudanças (SIQUEIRA; BOTELHO; COELHO, 2002; MORAIS; RODRIGUES; GERHARDT, 2008).

Durante o envelhecimento, ocorre à modificação da composição corporal, através da redução do teor de água, aumento de gordura e declínio da massa muscular esquelética. Além do declínio de mais de $15 \%$ do gasto metabólico basal, devido à redução do tecido magro, principalmente de células musculares metabolicamente ativas (SILVA et al., 2006). Ou seja, redução da massa muscular e aumento da gordura corporal (SANTOS et al., 2002).

Com o avançar da idade ocorre à redução da força e da potência do músculo, podendo influenciar na autonomia, bem-estar e qualidade de vida dos idosos. Além disso, a sarcopenia, que é a redução de fibras musculares e unidades motoras, contribui para outras alterações associadas à idade, como menor densidade óssea (PRADO et al., 2010). A flexibilidade também sofre modificações, declina e reduz sistemicamente o movimento ativo e passivo de todas as articulações (POLATO, 2010; EUHARDY, 2001).

A alimentação saudável e a prática regular de atividade física tem o poder de minimizar os efeitos deletérios que esse processo traz, além de garantir uma vida saudável e com autonomia por mais tempo, bem como aumentar a longevidade (SILVA JÚNIOR et al., 2011; BUSBY-
-WHITEHEAD, 2001). Exercícios físicos planejados, estruturados e repetitivos, como a musculação, melhoram ou mantem o nível da saúde do indivíduo, além de beneficiar o funcionamento de vários órgãos, na prevenção e tratamento de várias doenças e no desempenho das habilidades motoras (COELHO et al., 2011).

A prática contínua desses exercícios reduz os efeitos do envelhecimento do sistema muscular e contribui para o envelhecimento saudável (GLENN; MULCARE, 2005), uma vez que se percebe uma perda significativa de força e massa muscular em idosos (PRADO et al., 2010). Idosos fisicamente ativos apresentam mais massa muscular e uma maior aptidão física e funcional (FERNANDES, 2007; SOARES; ALABARSE, 2005).

Visando avaliar o quanto a prática regular de musculação interfere na composição corporal e na aptidão física, este estudo tem como objetivo descrever e relacionar a antropometria com a força muscular, a flexibilidade, a porcentagem de massa gorda, o tempo e a frequência semanal de treinos em idosos praticantes de musculação.

\section{Materiais e métodos}

Para responder aos questionamentos da pesquisa, optou-se pelo delineamento do estudo transversal, que segundo Rouquayrol (1994 apud BORDALO, 2006) é uma busca epidemiológica, em que causa e efeito são observados em um mesmo momento. Participaram do estudo 43 idosos praticantes de musculação do município de Ijuí(RS) selecionados de forma voluntária não probabilística. 
O estudo respeitou os princípios éticos e legais, de acordo com as recomendações das Diretrizes e Normas Regulamentadoras de Pesquisas, envolvendo seres humanos segundo a Resolução do Conselho Nacional de Saúde n. 196/96. O projeto foi submetido e aprovado pelo Comitê de Ética e Pesquisa da Universidade Regional do Noroeste do Estado do Rio Grande do Sul (Unijuí) (Parecer n. 196.1/2011).

Todos os participantes da pesquisa assinaram o Termo de Consentimento Livre e Esclarecido (TCLE). Para a coleta dos dados, buscou-se saber a idade, o tempo que frequenta a academia, a frequência semanal, utilizou-se um instrumento que investiga a presença de comorbidades, teste distância dedo-solo, senta-levanta, dinamometria e avaliação da composição corporal.

O teste distância dedo-solo (PATTENON et al., 2008) avaliou a flexibilidade dos participantes (os centímetros que ultrapassavam o ponto zero foram anotados como negativos). O teste senta-levanta, proposto pelo American College of Sports Medicine (2000), foi utilizado para mensurar a força dos membros inferiores.

A dinamometria foi empregada para aferir à força dos membros superiores, utilizando o Dinamômetro (Kratos Brasil). Esse teste aplicou-se a um voluntário mantendo ambos os braços estendidos no prolongamento do corpo, por meio de três verificações e, posteriormente, a média de cada membro (ILHA, 2009).

A Bioimpedância portátil, modelo HBF-306 (Omron), avaliou a composi- ção corporal, mensurando a porcentagem (\%) de massa gorda, como também o Índice de Massa Corporal (IMC). Os valores de IMC obtidos foram estratificados segundo Ministério da Saúde (BRASIL, 2006), em que os pontos de corte, estabelecidos para idosos, são $\leq$ $22 \mathrm{~kg} / \mathrm{m}^{2}$ para baixo peso, $>22$ e $<27$ $\mathrm{kg} / \mathrm{m}^{2}$ para eutrofia e $\geq 27 \mathrm{~kg} / \mathrm{m}^{2}$ para sobrepeso.

Os dados foram organizados em uma planilha no Microsoft Office Excel ${ }^{\circledR} 2010$ e realizada a análise estatística descritiva e analítica com o mesmo programa. Os dados serão apresentados em média \pm desvio padrão.

\section{Resultados}

Dos 43 voluntários, $37,20 \%$ (16) eram do gênero masculino e $62,80 \%$ (27) do gênero feminino, com média de idade de $65,84 \pm 4,76$ anos, visto que os homens apresentaram média de $67,18 \pm 5,16$ anos, e as mulheres $65,03 \pm 4,41$ anos.

O tempo médio de academia foi de $70,67 \pm 100,98$ meses, desses $44,87 \pm 51,97$ meses para os homens e $85,96 \pm 119,42$ meses para as mulheres. Já a frequência média dos treinos foi de $3,04 \pm 1,02$ vezes por semana, dos quais $3,43 \pm 1,26$ e $2,81 \pm 0,78$ vezes por semana para homens e mulheres, respectivamente.

A média da pressão arterial foi de $125 \pm 11,8 \mathrm{mmHg}$ e $80 \pm 8,16 \mathrm{mmHg}$ para a sistólica e diastólica, respectivamente. Já a média da frequência cardíaca em repouso foi de $76 \pm 10 \mathrm{bpm}$.

A hipertensão arterial sistêmica foi a comorbidade mais prevalente na amostra estudada, 48\% (21) dos voluntários 
apresentam essa patologia, enquanto $23,25 \%$ (dez) voluntários não apresentam nenhuma comorbidade.
A Tabela 1 apresenta a classificação do IMC dos participantes conforme o sexo masculino e feminino.

Tabela 1 - Classificação da amostra segundo o IMC

\begin{tabular}{lrrr}
\hline & \multicolumn{1}{c}{ Geral } & \multicolumn{1}{l}{ Homens } & \multicolumn{1}{c}{ Mulheres } \\
\hline Baixo peso & $4(9,30 \%)$ & $0(0,00 \%)$ & $4(9,30 \%)$ \\
Eutrofia & $19(44,18 \%)$ & $5(11,62 \%)$ & $14(32,55 \%)$ \\
Sobrepeso & $20(46,51 \%)$ & $11(25,58 \%)$ & $9(20,93 \%)$ \\
\hline
\end{tabular}

Fonte: Strassburger et al. ( 2010).

A maior parte dos idosos participantes da pesquisa está com sobrepeso. Os homens, na maioria, seguem esse resultado, enquanto a maior parte das mulheres apresenta peso adequado (eutrofia).
A caracterização da amostra conforme as variáveis (idade, tempo de academia, frequência, distância dedo-solo, $\%$ massa gorda e força dos membros), classificadas segundo o IMC são apresentadas na Tabela 2.

Tabela 2. Características dos idosos conforme o IMC

\begin{tabular}{|c|c|c|c|c|}
\hline & Média geral & Baixo peso & Eutrofia & Sobrepeso \\
\hline Idade (anos) & $65,83 \pm 4,76$ & $67,50 \pm 2,88$ & $65,10 \pm 4,99$ & $66,20 \pm 4,89$ \\
\hline Tempo de academia (meses) & $70,67 \pm 100,98$ & $84 \pm 41,59$ & $65,05 \pm 91,62$ & $73,35 \pm 119,33$ \\
\hline Frequência (vezes/semana) & $3,04 \pm 1,02$ & $3 \pm 0$ & $3,26 \pm 1,28$ & $2,85 \pm 0,81$ \\
\hline Distância dedo-solo (cm) & $1,10 \pm 8,88$ & $-1,87 \pm 5,92$ & $2,26 \pm 9,18$ & $0,6 \pm 9,25$ \\
\hline Massa gorda (\%) & $34,54 \pm 4,61$ & $30,86 \pm 4,00$ & $33,19 \pm 3,75$ & $36,31 \pm 4,83$ \\
\hline Força membro superior direito (kgf) & $28,38 \pm 9,06$ & $22,49 \pm 5,79$ & $27,15 \pm 8,47$ & $30,74 \pm 9,68$ \\
\hline Força membro superior esquerdo (kgf) & $25,84 \pm 8,16$ & $22,75 \pm 7,71$ & $24,72 \pm 6,84$ & $27,52 \pm 9,34$ \\
\hline Força membros inferiores (elevações) & $30,30 \pm 8,73$ & $26,5 \pm 6,85$ & $33,26 \pm 8,75$ & $28,25 \pm 8,50$ \\
\hline
\end{tabular}

Fonte: Strassburger et al. (2010).

Indivíduos classificados como baixo peso apresentam um tempo de academia maior, bem como uma melhor flexibilidade. Os eutróficos são os que apresentam uma maior frequência semanal de academia e menor flexibilidade. Em relação à força, os indivíduos com sobrepeso destacam-se na força dos membros superiores, enquanto os eutróficos na força dos membros inferiores. 
Tabela 3. Características dos idosos do sexo masculino conforme o IMC

\begin{tabular}{lrrr}
\hline & \multicolumn{1}{c}{ Média geral } & \multicolumn{1}{c}{ Eutrofia } & \multicolumn{1}{c}{ Sobrepeso } \\
\hline Idade (anos) & $67,18 \pm 5,16$ & $68,80 \pm 5,93$ & $66,45 \pm 4,90$ \\
Tempo de academia (meses) & $44,87 \pm 51,97$ & $60,40 \pm 73,88$ & $37,81 \pm 41,15$ \\
Frequência (vezes/semana) & $3,43 \pm 1,26$ & $4,20 \pm 1,64$ & $3,09 \pm 0,94$ \\
Distância dedo-solo (cm) & $5,56 \pm 11,50$ & $10,60 \pm 11,19$ & $3,27 \pm 11,39$ \\
Massa gorda (\%) & $31,38 \pm 2,83$ & $27,82 \pm 1,15$ & $32,67 \pm 2,00$ \\
Força membro superior direito (kgf) & $37,07 \pm 7,39$ & $36,45 \pm 8,51$ & $37,35 \pm 7,26$ \\
Força membro superior esquerdo (kgf) & $31,72 \pm 7,79$ & $29,65 \pm 5,75$ & $32,66 \pm 8,64$ \\
Força membros inferiores (elevações) & $32,18 \pm 8,15$ & $34,20 \pm 11,54$ & $31,27 \pm 6,60$ \\
\hline
\end{tabular}

Fonte: Strassburger et al. (2010).

A Tabela 3 apresenta a classificação dos homens conforme o IMC. Não foi encontrado nenhum homem com baixo peso na amostra estudada. Os que apresentam um maior tempo de academia e maior frequência semanal de treinos são os eutróficos, enquanto os com sobrepeso destacam-se na melhor flexibilidade. A força muscular é semelhante entre os grupos.

A caracterização das mulheres conforme o IMC é apresentado na Tabela 4.

Tabela 4. Características dos idosos do sexo feminino conforme o IMC

\begin{tabular}{|c|c|c|c|c|}
\hline & Média geral & Baixo peso & Eutrofia & Sobrepeso \\
\hline Idade (anos) & $65,03 \pm 4,41$ & $67,50 \pm 2,88$ & $63,78 \pm 4,07$ & $65,88 \pm 5,15$ \\
\hline Tempo de academia (meses) & $85,96 \pm 119,42$ & $84 \pm 41,56$ & $66,71 \pm 99,65$ & $116,77 \pm 166,86$ \\
\hline Frequência (vezes/semana) & $2,81 \pm 0,78$ & $3 \pm 0$ & $2,92 \pm 0,99$ & $2,55 \pm 0,52$ \\
\hline Distância dedo-solo (cm) & $-1,53 \pm 5,64$ & $-1,87 \pm 5,92$ & $-0,71 \pm 6,48$ & $-2,66 \pm 4,41$ \\
\hline Massa gorda (\%) & $36,36 \pm 4,48$ & $30,86 \pm 4,00$ & $34,72 \pm 2,59$ & $40,75 \pm 3,19$ \\
\hline Força membro superior direito (kgf) & $23,24 \pm 5,22$ & $22,49 \pm 5,79$ & $23,83 \pm 5,64$ & $22,64 \pm 4,81$ \\
\hline Força membro superior esquerdo (kgf) & $22,36 \pm 6,22$ & $22,75 \pm 7,71$ & $22,97 \pm 6,48$ & $21,24 \pm 5,76$ \\
\hline Força membros inferiores (elevações) & $29,18 \pm 9,02$ & $26,50 \pm 6,85$ & $32,92 \pm 8,04$ & $24,55 \pm 9,46$ \\
\hline
\end{tabular}

Fonte: Strassburger et al. (2010).

A força dos membros superiores é semelhante entre os grupos de mulheres, as eutróficas destacam-se na força dos membros inferiores. As mulheres com sobrepeso apresentam uma melhor flexibilidade e um maior tempo de academia. 


\section{Discussão}

Do total de idosos avaliados, a maioria, $62,80 \%$, são mulheres, as quais iniciam os cuidados com a sua saúde mais cedo, uma vez que a média de idade feminina é menor que a masculina. Isso reflete a importância que a mulher confere à prevenção de agravos da saúde.

Contudo, isso pode ser explicado, pois as mulheres durante a socialização recebem estímulos para reproduzirem e consolidarem os papéis que as tornam responsáveis pelos cuidados e prestação de serviços aos outros, o que não ocorre com os homens (GOMES; NASCIMENTO; ARAÚJO, 2007). Além do fato de que a expectativa de vida feminina sempre se mostrou maior que a masculina (MOREIRA et al., 2009).

A maioria das participantes encontra-se com sobrepeso $(46,51 \%)$, seguida da eutrofia (44,18\%), não se encontrou nenhum homem com baixo peso, a maioria desses encontra-se com sobrepeso, enquanto a maioria das mulheres são eutróficas.

A composição corporal das mulheres pode não se alterar com o treinamento físico. Conforme Silva Júnior (et al., 2011), em uma pesquisa com 34 mulheres entre cinquenta e 89 anos participantes de um grupo de atividade física, demonstraram que mesmo com o treinamento físico encontravam-se com excesso de peso.

Matsudo et al. (2002) compararam a evolução do perfil antropométrico de mulheres, entre cinquenta e 79 anos, ativas no período de um ano, de acordo com a idade cronológica encontrando sobrepeso, apesar da participação nos programas de exercícios aeróbios.
Indivíduos mais ativos apresentam baixo peso corporal no IMC e menor porcentagem de gordura (LONDERO, 2007), corroborando com a nossa pesquisa, provavelmente, porque esses indivíduos apresentam um maior tempo de academia. A maior frequência de treinos semanais é encontrada no grupo dos indivíduos eutróficos, demonstrando que a prática regular de exercícios físicos auxilia no controle do peso corporal, da força muscular e da pressão arterial (LINS; CORBUCCI, 2007).

A menor flexibilidade é encontrada nos eutróficos, ressaltando a importância da manutenção dessa para a facilitação dos movimentos realizados durante as atividades diárias e sua redução, que ocorre naturalmente durante $o$ envelhecimento, podendo ocasionar a perda parcial da independência dos movimentos (LONDERO, 2007).

A maior força muscular dos membros superiores é encontrada nos idosos com sobrepeso, enquanto a de membros inferiores nos eutróficos. Além de incrementar a força muscular, o treino de força permite aos idosos reverterem situações inusitadas de desequilíbrio (PRADO et al., 2010).

O tempo de academia dos homens, quando comparado com o tempo das mulheres, é menor, estando relacionado a níveis mais elevados de IMC e porcentagem de gordura. Porém, elas apresentam uma frequência semanal de treinos menor que a dos homens.

Com o avanço da idade, os indivíduos tendem a tornarem-se mais sedentários, reduzindo suas capacidades físicas. A redução das atividades físicas colabora para a aparição de doenças crônicas que 
contribuem para a deterioração do processo de envelhecimento (MOTTA, 2008).

Ambos, homens e mulheres, com sobrepeso apresentam uma menor frequência semanal de treinos, porém as mulheres têm maior tempo de academia. Esses fatores, associados com elevados percentuais de massa gorda, comprovam as modificações da composição corporal decorrente do envelhecimento. Nesse sentido, há que se destacar a redução do teor de água, aumento de gordura, declínio da massa muscular esquelética e de mais de $15 \%$ do gasto metabólico basal,devido à redução do tecido magro, principalmente das células musculares metabolicamente ativas (SILVA et al., 2006).

A maior frequência semanal de treinos das mulheres com baixo peso ocasiona uma menor porcentagem de massa gorda, corroborando que a atividade física encontra-se diretamente associada à composição corporal, uma vez que idosos ativos apresentam menores valores de massa de gordura e maiores de massa muscular que os inativos (SOARES; ALABARSE, 2005).

Nos homens eutróficos, esse fato também é encontrado, além de que, quando comparados aos idosos com sobrepeso, apresentam um maior tempo de prática dessas atividades. Espera-se, com a prática regular de exercícios físicos, que ocorra um aumento na qualidade de vida, redução do risco de quedas e manutenção e/ou melhora da função em diversas atividades (KAUFFMAN; JACKSON, 2001), deixando o idoso independente por mais tempo.

Ao avaliar os níveis de flexibilidade, percebe-se que as mulheres apresentam valores muito melhores que os homens, independentemente da sua classificação do IMC. Nem todos os idosos apresentam diminuição da flexibilidade à medida em que envelhecem, e o aumento na amplitude de movimento podem ser obtido por meio de exercícios físicos regulares e programas de alongamento (EUHARDY, 2001).

Dentre os homens, os com sobrepeso apresentam uma melhor flexibilidade, porém esses valores estão muito aquém dos valores encontrados nas mulheres. Isso indica as mulheres como portadoras de maiores níveis de flexibilidade quando comparadas aos homens (DIAS; GURJÃO; MARUCCI, 2006), destacando que a flexibilidade declina com a idade, porém em homens o declínio é maior (BELL; HOSHIZAKI, 1981).

A maior força muscular dos membros inferiores é encontrada nos eutróficos, tanto nos homens como nas mulheres. Exercícios com pesos livres produzem incrementos significativos na força muscular das extremidades corporais em idosos. Esses ganhos observados na força, ocorrem devido às adaptações neurais, também podem ser atribuídos ao incremento no tamanho da fibra muscular. Exercícios com pesos ocasionam a melhora significativa da velocidade de levantar-se da posição sentado e para subir escadas (RASO; MATSUDO; MATSUDO, 2001).

Quanto aos membros superiores, entre as mulheres, não foi encontrado diferença significativa dos valores entre os grupos. Já entre os homens, os participantes com sobrepeso apresentam maior força. Estímulos adequados de treino em idosos, independentemente do sexo, ten- 
dem a retardar a redução da massa muscular e da força que normalmente são/ estão associados com o envelhecimento (CARVALHO et al., 2004). Contudo, os homens são significativamente mais fortes que as mulheres, independente do grupo muscular avaliado.

\section{Conclusões}

Considerando os objetivos da pesquisa, os resultados encontrados indicam que, para o grupo de idosos avaliados, a prática de musculação não está interferindo na aptidão física e na antropometria, apresentando dificuldades em manter os idosos com a porcentagem de gordura e o IMC ideal. Porém, essa atividade traz consigo benefícios no que diz respeito à manutenção da flexibilidade $\mathrm{e}$ da força muscular, visto que as mulheres são mais beneficiadas com o primeiro e os homens com o segundo aspecto citado.

Percebe-se também, que esse é um meio de socialização para a população de idosos, principalmente, quando considerado o tempo em que frequenta a academia e a frequência semanal das atividades desenvolvidas.

Essa modalidade de exercício físico é procurada de forma crescente pelos idosos, para manter e/ou melhorar suas condições físicas. Nesse sentido, outros estudos devem ser realizados a respeitodesse assunto, sobretudo no que se refere à relação do exercício físico, hábitos alimentares, aptidão física e composição corporal, para melhor compreender os efeitos da prática de musculação na aptidão física de idosos.
Relationship of body composition and physical fitness of older bodybuilders

\section{Abstract}

The Brazilian population is aging and exercise has much to contribute, keeping the body healthy and reducing the deleterious effects of aging. The aim of the study was to investigate the anthropometric and physical performance in elderly individuals practicing bodybuilding. This is a cross-sectional study, which presents and discusses the findings of the evaluation of 43 elderly, bodybuilders, regarding body composition, flexibility and strength of upper and lower limbs. The average time the gym was 70.67 \pm 100.98 months, a weekly frequency of $3.04 \pm 1.02$ times. Overweight was most frequently found in $46.51 \%$ of the elderly, being more frequent in men. Women have better results in flexibility, while men show better results for strength tests. In general, the time of gym does not interfere in body composition, but the overweight men have shorter gym, while overweight women have longer gym. It follows that the group rated bodybuilding is not providing great changes in physical fitness and anthropometry. It is noted that this modality is increasingly being spread by this population, showing the need for more studies in the area for the perception of the real effects of such training in the aging process.

Keywords: Exercise. Anthropometry. Aging.

\section{Referências}

AMERICAN COLLEGE OF SPORTS MEDICINE (ACSM). Manual do ACSM para teste de esforço e prescrição de exercício. Rio de Janeiro: Revinter, 2000.

BORDALO, A. A. Estudo transversal e/ou longitudinal. Revista Paraense de Medicina, Belém, v. 20, n. 4, dez. 2006. 
BELL, R. D.; HOSHIZAKI, T. B. Relationships of age and sex with range of motion of seventeen joint actions in humans. Canadian. Journal of Applied Sports and Sciences, Toronto, v. 6, p. 202-206, 1981.

BRASIL, Ministério da Saúde (MS). Envelhecimento e saúde da pessoa idosa. Brasília: MS/Departamento de Atenção Básica, 2006, p. $37-40$ e $148-167$.

BUSBY-WHITEHEAD, J. Exercícios físicos para idosos. In: GALLO, J. J. et al. Reichel assistência ao idoso: aspectos clínicos do envelhecimento. Rio de Janeiro: Guanabara Koogan, 2001. p. 109-113.

CARVALHO, J. et al. Força muscular em idosos II: efeito de um programa complementar de treino na força muscular de idosos de ambos os sexos. Revista Portuguesa de Ciências do Desporto, Porto, v. 4, n. 1, p. 58-65, 2004.

COELHO, M. A. G. et. al. Perfil de idosos do município de Itaúma/MG e influencia da atividade física na dor crônica e na capacidade funcional. Fisioterapia Brasil, cidade, v. 12, n. 2, p. 94-99, 2011.

DIAS, R. M. R.; GURJÃO, A. L. D.; MARUCCI, M. F. N. Benefícios do treinamento com pesos para aptidão física de idosos. Acta Fisiatrica, São Paulo, v. 13, n. 2, p. 90-95, 2006.

EUHARDY, R. Contraturas. In: KAUFFMAN, T. L. Manual de reabilitação geriátrica, Rio de Janeiro: Guanabara Koogan, 2001, p. 63-67.

FERNANDES, G. T. Análise de um programa de caminhada na força muscular em idosos. Porto, Faculdade de Desporto, Universidade do Porto, 2007.

GLENN, T. M.; MULCARE, J. A. Princípios do exercício aeróbico. In: KISNER, C.;COLBY, L. A. Exercícios terapêuticos: fundamentos e técnicas. Barueri: Manole, 2005. p. 149-170.

GOMES, R.; NASCIMENTO, E. F.; ARAÚJO, F. C. Por que os homens buscam menos os serviços de saúde do que as mulheres? As ex- plicações de homens com baixa escolaridade e homens com ensino superior. Cadernos de Saúde Pública, Rio de Janeiro, v. 23, n. 3, p. 565-574, 2007.

ILHA, F. V. Análise dinamométrica em membros superiores em praticantes de musculação na academia do SESC. Cruz Alta : Universidade de Cruz Alta, 2009.

SILVA JÚNIOR, J. P. et al. Estabilidade das variáveis de aptidão física e capacidade funcional de mulheres fisicamente ativas de 50 a 89 anos. Revista Brasileira de Cineantropometria e Desempenho Humano, Florianópolis, v. 13, n. 1, p. 8-14, 2011.

KAUFFMAN, T. L.; JACKSON, O. O indivíduo como um todo. In: KAUFFMAN, T. L. Manual de reabilitação geriátrica. Rio de Janeiro: Guanabara Koogan, 2001. p. 3-7.

LINS, R. G.; CORBUCCI, P. R. A importância da motivação na pratica de atividade física para idosos. Estação Científica Online, Juíz de Fora, n. 4, p. 1-13, abr./maio 2007.

LONDERO, E. B. A prática de atividade física na terceira idade. Santa Rosa: Universidade Regional do Noroeste do Estado do Rio Grande do Sul, 2007.

MATSUDO, S. M.; NETO, T. L. B.; MATSUDO, V. K. R. Perfil antropométrico de mulheres maiores de 50 anos, fisicamente ativas, de acordo com a idade cronológica: evolução de 1 ano. Revista Brasileira de Ciências e Movimento, Brasília, v. 10, n. 2, p. 15-26, 2002.

MORAIS, E. P.; RODRIGUES, R. A. P.; GERHARDT, T. E. Os idosos mais velhos no meio rural: realidade de vida e saúde de uma população do interior gaúcho. Texto Contexto - Enfermagem, Florianópolis, v. 17, n. 2, p. 374-383, 2008.

MOREIRA, A. J. et. al. Composição corporal de idosos segundo a antropometria. Revista Brasileira de Geriatria e Gerontologia, Rio de Janeiro, v. 12, n. 2, p. 201-213, 2009. 
MOTTA, A. M. Associação entre fenótipos musculares e variáveis de aptidão física aeróbia em mulheres idosas. 2008. Dissertação (Mestrado em Educação Física) - Universidade Católica de Brasília, Brasília, 2008.

PATTENON, R. et al. Adaptação funcional do aparelho respiratório e da postura no idoso. Revista Brasileira de Ciências do Envelhecimento Humano, Passo Fundo, v. 5, n. 2, p. 64-77, jul./dez. 2008.

POLATO, D. Avaliação objetiva do tônus muscular em idosos praticantes de atividade física. 2010. Dissertação (Mestrado em Engenharia Biomédica) - Universidade Federal do Rio de Janeiro, Rio de Janeiro, 2010.

PRADO, R. A. et al. A influência de exercícios resistidos no equilíbrio, mobilidade funcional e na qualidade de vida de idosas. $O$ mundo da Saúde, São Paulo, v. 34, n. 2, p. 183-191, 2010.

RASO, V.; MATSUDO, S. M. M.; MATSUDO, V. K. R. A força muscular de mulheres idosas decresce principalmente após oito semanas de interrupção de um programa de exercícios com pesos livres. Revista Brasileira de Medicina do Esporte, São Paulo, v. 7, n. 6, p. 177-186, nov./dez. 2001.

SANTOS, A. E. et al. Treinamento de força e potência para idosos. Revista Digital Vida Saúde, v. 1, n. 3, 2002. Disponível em: <http:// www.revistadigitalvidaesaude.hpg.com.br/ artv1n3_09.pdf>. Acesso em: 2 mar. 2011.

SILVA, T. A. A. et al. Sarcopenia associada ao envelhecimento: aspectos etiológicos e opções terapêuticas. Revista Brasileira de Reumatologia, São Paulo, v. 46, n. 6, p. 391-397, 2006.

SIQUEIRA, R. L.; BOTELHO, M. I. V.; COELHO, F. M. G. A velhice: algumas considerações teóricas e conceituais. Ciência \& Saúde Coletiva, Rio de Janeiro, v. 7, n. 4, p. 899-906, 2002.

SOARES, J.; ALABARSE, S. Envelhecimento e atividade física. In: RAMOS, L. R.; NETO, J. T. Geriatria e gerontologia, Barueri: Manole, 2005. p. 255-270.
VERAS, R. Envelhecimento populacional contemporâneo: demandas, desafios e inovações. Revista de Saúde Pública, São Paulo, v. 43, n. 3, p. 548-554, 2009.

Em busca de uma assistência adequada à saúde do idoso: revisão da literatura e aplicação de um instrumento de detecção precoce e de previsibilidade de agravos. Cadernos de Saúde Pública, Rio de Janeiro, v. 19, n. 3, p. 705-715, 2003. 